\title{
Early onset of severe lymphopenia during definitive radiotherapy correlates with mean body dose and predicts poor survival in cervical cancer
}

\author{
Li Yang ${ }^{\mathrm{a}, \mathrm{b}}$, Zhiyuan Xu ${ }^{\mathrm{b}, *}$, Lingyu Ma ${ }^{\mathrm{b}}$, Qin Liu ${ }^{\mathrm{b}}$, Amy T.Y. Chang ${ }^{\mathrm{b}, \mathrm{c}}$, Qian Wang ${ }^{\mathrm{b}}$, Jiandong Zha ${ }^{\mathrm{b}}$, \\ Jinliang Zhang ${ }^{\mathrm{b}}$, Xiaoqin Jiang ${ }^{\mathrm{b}}$, Jingjing Zhang ${ }^{\mathrm{b}}$, Feng-Ming (Spring) Kong ${ }^{\mathrm{b}, \mathrm{d}}$ and Linlang Guo ${ }^{\mathrm{a}, *}$ \\ ${ }^{a}$ Department of Pathology, Zhujiang Hospital, Southern Medical University, Guangzhou, Guangdong, China \\ ${ }^{\mathrm{b}}$ Clinical Oncology Center, the University of Hong Kong - Shenzhen Hospital, Shenzhen, Guangdong, China \\ ${ }^{\mathrm{c}}$ Hong Kong Sanatorium and Hospital, Hong Kong, China \\ ${ }^{\mathrm{d}}$ The University of Hong Kong Li Ka Shing Medical School, Hong Kong, China
}

Received 24 May 2021

Accepted 5 January 2022

\begin{abstract}
.
BACKGROUND: Lymphopenia during definitive radiotherapy (RT) has been shown to reduce survival in patients with cervical cancer. However, there are few studies on the significance of onset time of lymphopenia during RT in patients with cervical cancer. OBJECTIVE: This study aimed to exam the prognostic significance of early onset of severe lymphopenia (EOSL) during definitive RT in patients with cervical cancer.

METHODS: Newly diagnosed cervical cancer patients treated with definitive RT from January 2015 to December 2019 were eligible for this retrospective study. EOSL was defined as first onset of grade 3-4 lymphopenia $\leqslant 3$ weeks from the start of RT. Mean body dose (MBD) was the mean radiation dose absorbed by the body during the whole course of external beam RT (EBRT) and was directly obtained from the dose volume histogram (DVH) of the EBRT planning. Logistic regression analysis and restricted cubic spline (RCS) models were applied to assess relationships between clinicopathological factors and EOSL. Survival analysis was performed using Kaplan-Meier curves and log-rank test. A COX regression model was developed to predict overall survival (OS).

RESULTS: A total of 104 patients were included and 59.6\% had EOSL. MBD $(P=0.04)$, concurrent cisplatin $(P=0.011)$, and pre-RT absolute lymphocyte count (ALC) $(P=0.001)$ were associated with EOSL. A linear relationship $(P$ for non-linearity $=$ $0.803)$ between MBD and risk of EOSL was found. Patients with EOSL had decreased OS (2-yr $75.1 \%$ vs $91.1 \%, P=0.021)$ and progression-free survival (PFS) (2-yr $71.2 \%$ vs $83.7 \%, P=0.071$ ). An OS prediction COX model was developed with C-index of 0.835 and AUC of 0.872 .
\end{abstract}

CONCLUSIONS: EOSL during definitive RT correlates with MBD and predicts poor survival in patients with cervical cancer.

Keywords: Cervical cancer, definitive radiotherapy, early onset of severe lymphopenia, mean body dose, overall survival

\section{Introduction}

${ }^{*}$ Corresponding authors: Linlang Guo, Department of Pathology, Zhujiang Hospital, Southern Medical University, No. 253, Gongye Avenue, Guangzhou, Guangdong 510282, China. Tel.: +86 020 61643888; E-mail: linlangg@yahoo.com. Zhiyuan Xu, Clinical Oncology Center, the University of Hong Kong - Shenzhen Hospital, No. 1, Haiyuan $1^{\text {st }}$ Road, Futian District, Shenzhen, Guangdong 518053, China. Tel.: +86 075586913333 2107; E-mail: Xuzy@hkuszh.org.
Cervical cancer is one of the most common malignant tumors in women [1]. Definitive concurrent chemoradiotherapy (CCRT) is the preferred treatment for patients with localized cervical cancer who are not amenable to surgery [2]. However, there is still room for improvement in the prognosis of cervical cancer 
with a reported 5-year disease-free survival of less than $70 \%$ [3]. It is important to study risk factors for survival to guide personized treatment.

Immune system plays an important role in the development of malignant tumors [4] and is also essential for tumor response and eradication [5,6]. Lymphocytes have been recognized as major actors in the fight against tumor progression. Lymphopenia is a common side effect of anti-cancer treatment and has been reported to be an independent prognostic factor for poor survival in several cancer types [7]. The incidence rate of grade 3-4 lymphopenia during CCRT was as high as $89 \%$ in cervical cancer [8]. Previous studies showed that pre-, during-, and post-treatment lymphopenia may be associated with decreased survival in patients with locally advanced cervical cancer [8-10].

The onset time of lymphopenia during radiotherapy (RT) was clinically relevant in patients with lung cancer [11]: the median overall survival (OS) and progression-free survival (PFS) for patients with low absolute lymphocyte count (ALC) $\left(\leqslant 900\right.$ cells $\left./ \mathrm{mm}^{3}\right)$ at week 2 of RT were 15.3 and 5.9 months, respectively, compared to 18.9 and 12.5 months for patients with higher ALC $\left(>900\right.$ cells $\left./ \mathrm{mm}^{3}\right)(P=0.035$ and 0.053 , respectively) [11]. However, the prognostic value of onset time of severe lymphopenia during RT has not been fully addressed in cervical cancer. This study aimed to investigate the prognostic significance of incidence time of severe lymphopenia during definitive RT in patients with cervical cancer. The results of the study may provide references for clinicians in the process of making treatment decision.

\section{Methods}

\subsection{Patient selection}

This study conforms with The Code of Ethics of the World Medical Association (Declaration of Helsinki) and was approved by institutional ethics committee of the University of Hong Kong-Shenzhen Hospital (NO: [2019]049). Individual consent from each patient for this retrospective analysis was waived. Newly diagnosed cervical cancer patients treated with definitive RT from January 2015 to December 2019 were identified from patient database of the department $(N=$ 116). The process for selecting patients was presented in Fig. 1. Patients were eligible for this study if they met the following criteria: 1) $\geqslant 18$ years old, 2) Eastern Cooperative Oncology Group (ECOG) performance status $\leqslant 2,3)$ pathology-confirmed cervical squamous cell carcinoma, adenocarcinoma, or adenosquamous cell carcinoma, 4) International Federation of Gynecology and Obstetrics (FIGO) (2018) stage IB1-IVA, 5) treated with external beam radiotherapy (EBRT) followed by brachytherapy with or without concurrent cisplatin or induction chemotherapy (IC), 6) had complete blood count (CBC) tested before and weekly during RT. Patients were excluded if they had acquired immune deficiency syndrome (AIDS), PS $>2$, concomitant secondary primary malignant tumor, concurrent carboplatin, RT for adjuvant treatment or recurrent disease, or did not complete RT. All patients provided written informed consent form for the use of personal medical data for academic research purpose before treatment.

\subsection{Investigations, treatment and follow up}

According to the practice guideline of the department, all patients had gynecological physical examination by experienced gynecologists, contrast computed tomography (CT) of thorax, abdomen and pelvis or positron emission tomography (PET)-CT, and contrast magnetic resonance imaging (MRI) of pelvis (if no contraindication of MRI) for baseline staging. Cystoscopy or sigmoidoscopy were indicated only if bladder or rectum invasion was suspected. Patients also had serial CBC, liver and renal function tests before and weekly during RT. Patients received EBRT followed by brachytherapy with or without concurrent cisplatin or IC. Two types of EBRT techniques were used namely RapidArc or three-dimensional conformal radiotherapy (3D-CRT). GTV (Gross Tumor Volume)-T: the primary tumor from hyper intense signal of T2-weighted MRI images. GTV-N: locoregional pathologically enlarged lymph nodes. CTV (Clinical Target Volume)$\mathrm{T}$ included cervix, parametrium bilaterally, uterus and part of vagina. CTV-N: GTV-N $+3 \mathrm{~mm}$ margin. CTV$\mathrm{E}$ included regional lymphatics (common iliac, external iliac, internal iliac, presacral \pm para-aortic or inguinal). ITV (Internal Target Volume)-T: CTV-T + margins (10 $\mathrm{mm}$ anterior-posterior, $10 \mathrm{~mm}$ superiorinferior, $5 \mathrm{~mm}$ lateral). ITV45 = ITV-T + CTV-N + CTV-E. PTV (Planning Target Volume) 45: ITV45 $+5 \mathrm{~mm}$ margin. PTV-N: CTV-N $+5 \mathrm{~mm}$ margin. Dose/fractionation: PTV45: 1.8 Gy/Fr daily, 5 Fr/week up to $45 \mathrm{~Gy}$; PTV-N: using simultaneous integrated boost (SIB): $2.2 \mathrm{~Gy} / \mathrm{Fr}$ daily, $5 \mathrm{Fr} /$ week up to $55 \mathrm{~Gy}$ if lymph node was located within the pelvis, or $2.3 \mathrm{~Gy} / \mathrm{Fr}$ daily, $5 \mathrm{Fr} /$ week up to $57.5 \mathrm{~Gy}$ if lymph node was located outside the pelvis. 3D-CRT was divided into 2 


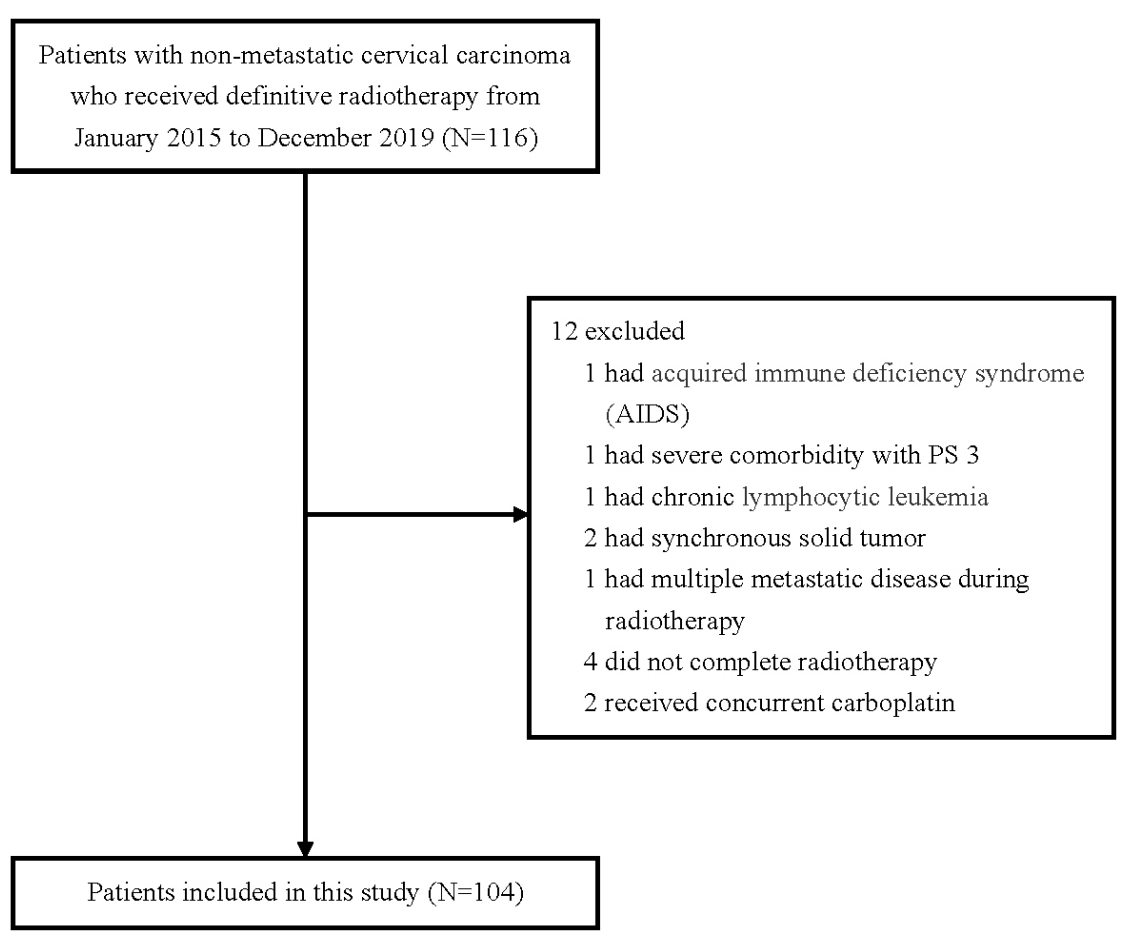

Fig. 1. Selection process of study population.

phases: phase I PTV45, 45 Gy in 25 fractions to pelvis; phase II pelvic wall boost, FIGO IIIB 16 Gy in 8 fractions, other stages $10 \mathrm{~Gy}$ in 5 fractions, $5 \mathrm{Fr} /$ week.

Brachytherapy was guided by CT or MRI and was started 4-5 weeks after the start of EBRT, ${ }^{192} \mathrm{Ir}$ (iridium) high dose rate with $6 \mathrm{~Gy} \times 4$ to point $\mathrm{A}$ once a week for 4 weeks were applied. Point A dose was aimed at cumulative equivalent dose in $2 \mathrm{~Gy} / \mathrm{Fr}$ (EQD2) of 80-85 Gy.

Concurrent cisplatin was applied at $40 \mathrm{mg} / \mathrm{m}^{2} \mathrm{ev}-$ ery week for up to 5-6 weeks. If waiting time for RT was more than 1 month, IC with paclitaxel plus carboplatin could be given at the discretion of the treating physicians.

After completion of RT, patients were followed up every 3 to 4 months for the first 2 years, every 4 to 6 months for third to fifth year and annually thereafter. Surveillance evaluations included physical examination, tumor marker (optional), imaging studies (optional) such as pelvic MRI, CT chest and abdomen or PET-CT, and biopsy if clinically indicated on follow up.

\subsection{Data collection}

Variables of interest in this study included patient demographics, clinicopathologic findings, and treatment related data. All these data were collected from hospital's electronic medical records. Common Terminology Criteria for Adverse Events (CTCAE) v4.03 was used to grade the myelosuppression. Grade 3 lymphopenia was defined as ALC $<0.5-0.2 \times 10^{9} / \mathrm{L}$ and grade 4 lymphopenia was defined as ALC $<0.2 \times 10^{9} / \mathrm{L}$. Mean body dose (MBD) was the mean radiation dose absorbed by the body within the range of CT simulation scan during the whole course of EBRT and was directly obtained from the dose volume histogram (DVH) of the EBRT plan in the Varian eclipse treatment planning system (version 15.0).

\subsection{Statistical analysis}

Data was analyzed using SPSS version 25.0 (SPSS, Inc., Chicago, IL, USA) statistical software and R version 3.6.1(R Core Team, Vienna, Austria). Demographics, clinicopathologic findings, and treatment related characteristics were described as median (interquartile range $[\mathrm{IQR}]$ ) values of continuous variables and percentages for categorical variables. Means between groups were tested for normal distribution with ShapiroWilk tests followed by either a student's $t$-test or a Wilcoxon rank-sum test. Early onset of severe lymphopenia (EOSL) was defined as first onset of grade 3 
4 lymphopenia $\leqslant 3$ weeks from the start of RT. A multivariate logistic regression model was used to identify factors associated with EOSL. Dose-response relationship between MBD and risk of EOSL was assessed by restricted cubic spline (RCS) functions. PFS was calculated from the start of RT to the first appearance of progressive disease or death from any cause. OS was calculated from the start of RT until the date of death from any cause or the last confirmed date of survival. Survival analysis was performed using the Kaplan-Meier curves, significance was tested with the log-rank test. Univariate and multivariate survival analyses were performed using Cox regression model. The performance of Cox regression model was evaluated using the Cindex and area under the curve (AUC) statistics. All $P$ values were two-sided and $<0.05$ was considered statistically significant.

\section{Results}

\subsection{Patient characteristics}

A total of 104 women were included in this study. The median age was 52.5 years. The FIGO (2018) stage distribution was $3(2.9 \%), 31(29.8 \%), 66(63.5 \%)$, and $4(3.8 \%)$ for stage I, II, III and IVA, respectively. One hundred and one $(97.1 \%)$ had squamous cell carcinoma. Seventeen (16.3\%) patients received IC before RT. Fifteen $(14.4 \%)$ patients had RT alone. The demographics, clinicopathologic findings, and treatment characteristics of the patients were listed in Table 1.

\subsection{Pattern and characteristics of lymphopenia and potential contributing factors of early onset of severe lymphopenia during $R T$}

ALC of all patients significantly declined during RT and generally recovered to some extent at the end of RT (Wilcoxon test, all $P$ values $\leqslant 0.0001$ ). (Figure 2) The values (median [IQR]) of pre-RT ALC, mid-RT ALC nadir, and end-RT ALC was $1.74(1.32-2.05) \times$ $10^{9} / \mathrm{L}, 0.22(0.17-0.29) \times 10^{9} / \mathrm{L}$, and $0.54(0.36-0.84)$ $\times 10^{9} / \mathrm{L}$, respectively. The median (IQR) time of first onset of severe lymphopenia (FOSL) from the start of RT was 20 (14-27) days (approximated to 3 weeks). The number (percentage) of patients with FOSL at the $1^{\text {st }}$ to $7^{\text {th }}$ week was $5(4.8 \%), 23(22.1 \%), 34(32.7 \%)$, $21(20.2 \%), 7(6.7 \%), 7(6.7 \%)$ and $2(1.9 \%)$, respectively (Fig. 3). The rest $5(4.8 \%)$ patients had grade 2 lymphopenia during RT (Fig. 3). Sixty-two (59.6\%) patients had EOSL.

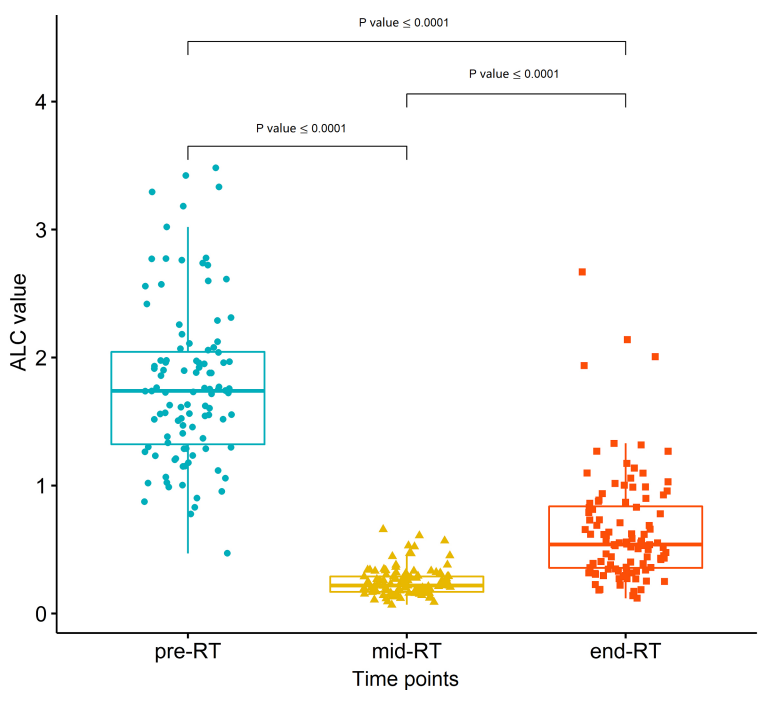

Fig. 2. The change and comparison of absolute lymphocyte count (ALC) at different time points (pre- RT, mid-RT, and end-RT). The values (median, interquartile range) of pre-RT ALC, mid-RT ALC nadir, and end-RT ALC were $1.74(1.32-2.05) \times 10^{9} / \mathrm{L}, 0.22$ $(0.17-0.29) \times 10^{9} / \mathrm{L}$, and $0.54(0.36-0.84) \times 10^{9} / \mathrm{L}$, respectively; Wilcoxon test, all $P$ values $\leqslant 0.0001$.

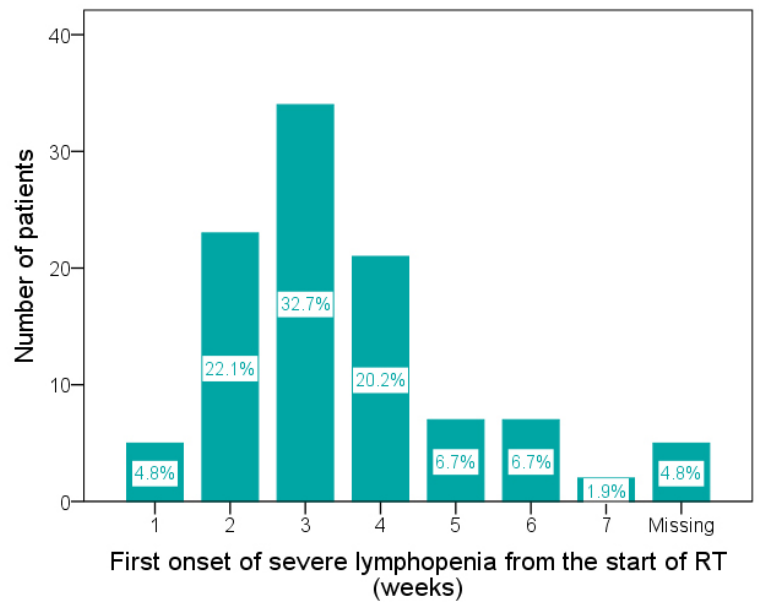

Fig. 3. Distribution of the time of first onset of severe lymphopenia (FOSL) from the start of radiotherapy (RT) (median 20 days, interquartile range 14-27 days). The number (percentage) of patients with FOSL at the $1^{\text {st }}$ to $7^{\text {th }}$ week was $5(4.8 \%), 23(22.1 \%), 34$ $(32.7 \%), 21(20.2 \%), 7(6.7 \%), 7(6.7 \%)$, and $2(1.9 \%)$, respectively. Five (4.8\%) patients with no grade 3-4 lymphopenia during RT was presented as missing data.

On univariate logistic regression analysis, higher MBD $(P=0.02)$, age $\leqslant 60$ years $(P=0.003)$, FIGO IIIA-IVA $(P=0.027)$, concurrent cisplatin $(P=$ $0.009)$, lower pre-RT hemoglobin (HGB) $(P=0.039)$, and lower pre-RT ALC $(P=0.001)$ were associated with EOSL. Multivariate logistic regression with back- 
Table 1

Patient characteristics and clinical outcomes

\begin{tabular}{|c|c|}
\hline Features & No. $(\%)$ or Median (IQR) \\
\hline Age & $52.5(46-63)$ \\
\hline$\leqslant 60$ & $72(69.2)$ \\
\hline$>60$ & $32(30.8)$ \\
\hline \multicolumn{2}{|l|}{ ECOG } \\
\hline $0-1$ & $90(86.5)$ \\
\hline 2 & $14(13.5)$ \\
\hline \multicolumn{2}{|l|}{ FIGO stage (2018) } \\
\hline $\mathrm{I}$ & $3(2.9)$ \\
\hline II & $31(29.8)$ \\
\hline III & $66(63.5)$ \\
\hline IVA & $4(3.8)$ \\
\hline \multicolumn{2}{|l|}{ Pathology } \\
\hline Squamous cell carcinoma & $101(97.1)$ \\
\hline Adenocarcinoma & $2(1.9)$ \\
\hline Adenosquamous cell carcinoma & $1(1.0)$ \\
\hline Body mass index & $23.1(20.1-24.9)$ \\
\hline \multicolumn{2}{|l|}{ Induction chemotherapy } \\
\hline Yes & $17(16.3)$ \\
\hline No & $87(83.7)$ \\
\hline \multicolumn{2}{|l|}{ Concurrent cisplatin } \\
\hline Yes & $89(85.6)$ \\
\hline No & $15(14.4)$ \\
\hline Concurrent cisplatin cycles & $5(3.75-6)$ \\
\hline \multicolumn{2}{|l|}{ EBRT technique } \\
\hline 3D-CRT & $27(26.0)$ \\
\hline RapidArc & $77(74.0)$ \\
\hline Mean body dose & $12.38(10.52-14.33)$ \\
\hline Pre-RT WBC (cells × $\left.10^{9} / \mathrm{L}\right)$ & $6.77(5.45-8.12)$ \\
\hline Pre-RT ANC (cells × $\left.10^{9} / \mathrm{L}\right)$ & $4.41(3.36-5.57)$ \\
\hline Pre-RT ALC (cells $\left.\times 10^{9} / \mathrm{L}\right)$ & $1.74(1.32-2.05)$ \\
\hline Pre-RT PLT (cells $\left.\times 10^{9} / \mathrm{L}\right)$ & $268(217.5-324.3)$ \\
\hline Pre-RT AMC $\left(\right.$ cells $\left.\times 10^{9} / \mathrm{L}\right)$ & $0.34(0.26-0.43)$ \\
\hline Pre-RT HGB (g/L) & $119(103-131)$ \\
\hline During-RT ALC nadir (cells $\times 10^{9} / \mathrm{L}$ ) & $0.22(0.17-0.29)$ \\
\hline \multicolumn{2}{|l|}{ Grade of lymphopenia during RT } \\
\hline 1 & $0(0.0)$ \\
\hline 2 & $5(4.8)$ \\
\hline 3 & $64(61.5)$ \\
\hline 4 & $35(33.7)$ \\
\hline \multicolumn{2}{|l|}{ Early onset of severe lymphopenia } \\
\hline Yes & $62(59.6)$ \\
\hline No & $42(40.4)$ \\
\hline \multicolumn{2}{|l|}{ Post-RT local residual or recurrent disease } \\
\hline Yes & $12(11.5)$ \\
\hline No & $92(88.5)$ \\
\hline \multicolumn{2}{|l|}{ Post-RT regional lymph nodes metastases } \\
\hline Yes & $5(4.8)$ \\
\hline No & $99(95.2)$ \\
\hline \multicolumn{2}{|l|}{ Post-RT distant metastases } \\
\hline Yes & $15(14.4)$ \\
\hline No & $89(85.6)$ \\
\hline \multicolumn{2}{|l|}{ Disease progression } \\
\hline Yes & $26(25)$ \\
\hline No & $78(75)$ \\
\hline \multicolumn{2}{|l|}{ Death } \\
\hline Yes & $22(21.2)$ \\
\hline No & $82(78.8)$ \\
\hline
\end{tabular}

IQR, interquartile range; ECOG, Eastern Cooperative Oncology Group; FIGO, Federation of Gynecology and Obstetrics; EBRT, external beam radiotherapy; 3D-CRT, three-dimensional conformal radiotherapy; WBC, white blood cell; ANC, absolute neutrophil count; ALC, absolute lymphocyte count; PLT, platelet; AMC, absolute monocyte count; HGB, hemoglobin; RT, radiotherapy. 


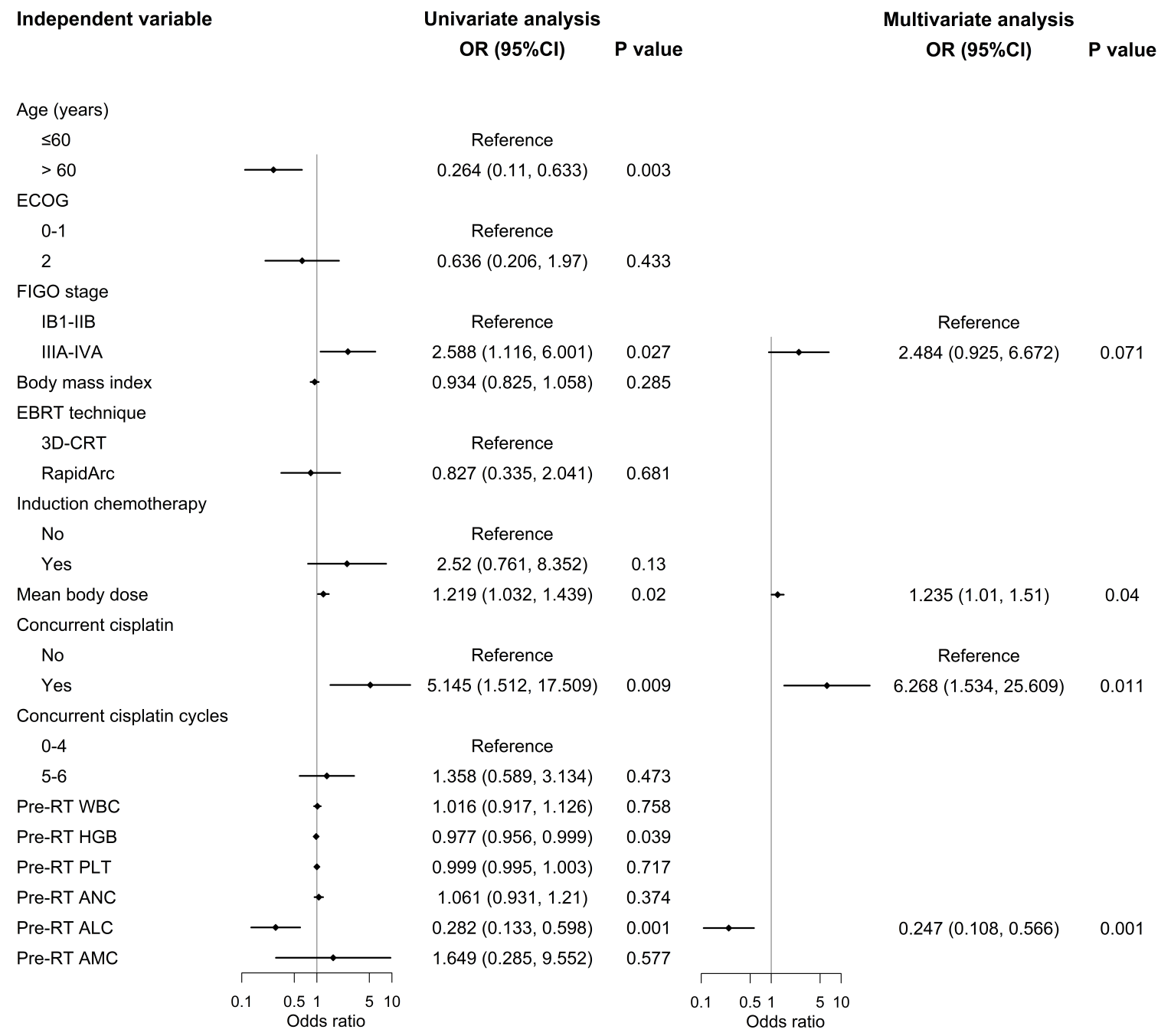

Fig. 4. Forest plot of univariate and multivariate logistic regression model for early onset of severe lymphopenia (EOSL). (ECOG, Eastern Cooperative Oncology Group; FIGO, Federation of Gynecology and Obstetrics; EBRT, external beam radiotherapy; 3D-CRT, three-dimensional conformal radiotherapy; RT, radiotherapy; WBC, white blood cell; HGB, hemoglobin; PLT, platelet; ANC, absolute neutrophil count; ALC, absolute lymphocyte count; AMC, absolute monocyte count; OR, odds ratio; CI, confidence interval.)

ward selection models was used to identify independent factors associated with EOSL. Factors kept in the multivariate model were based on the Akaike information criterion (AIC). The variables initially included in the model were treated as continuous variables (MBD, pre-RT HGB, and pre-RT ALC) and as categorical variables (age, FIGO stage, and concurrent cisplatin). MBD (Odds ratio [OR] 1.235, 95\% confidence interval [CI] $1.01-1.51, P=0.04)$, pre-RT ALC (OR $0.247,95 \% \mathrm{CI}$ $0.108-0.566, P=0.001$ ), concurrent cisplatin (OR $6.268,95 \%$ CI $1.534-25.609, P=0.011$ ) were correlated with EOSL in the final multivariate model. FIGO stage (OR 2.484, 95\% CI 0.925-6.672, $P=0.071$ ) was correlated with EOSL with borderline significance. Age and pre-RT HGB were not included in the final multivariate model (Fig. 4).

\subsection{Correlation between mean body dose (MBD) and risk of early onset of severe lymphopenia (EOSL)}

Patients with EOSL had higher MBD (mean \pm standard deviation [SD], $13.2 \pm 2.8 \mathrm{~Gy}, N=62$ ) than those without EOSL (11.9 $\pm 2.4 \mathrm{~Gy}, N=42 ; P=0.016$ ) (Fig. 5). The restricted cubic spline (RCS) function was applied to explore the dose-response relationship between MBD and risk of EOSL. We found a linear relationship $(P$ for nonlinearity $=0.803$ ) between MBD 


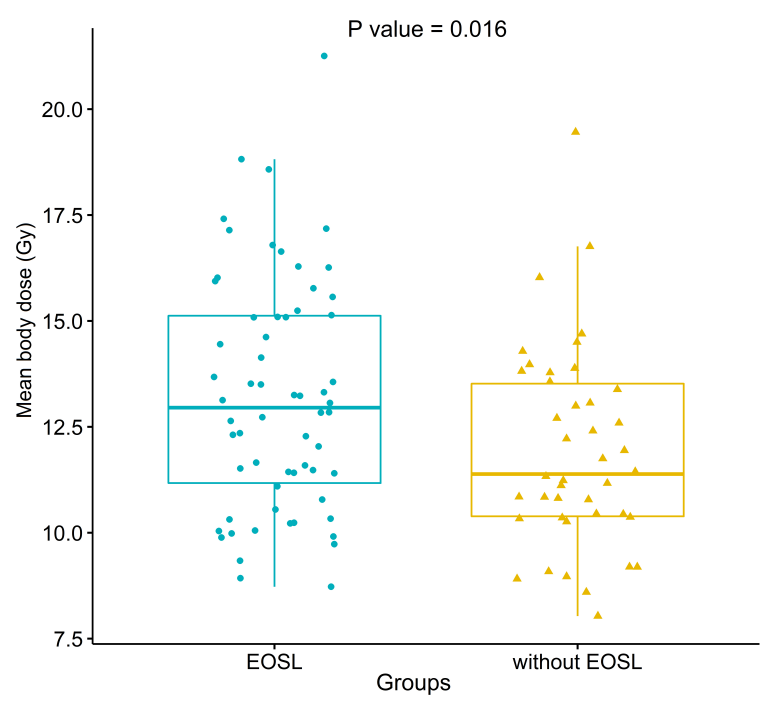

Fig. 5. The comparison of mean body dose (MBD) between patients with or without early onset of severe lymphopenia (EOSL). Patients with EOSL had higher MBD (mean \pm standard deviation, $13.2 \pm$ $2.8 \mathrm{~Gy}, N=62)$ than those without EOSL $(11.9 \pm 2.4 \mathrm{~Gy}, N=42$; $P=0.016)$.

and risk of EOSL (Fig. 6) According to the RCS curve after adjusting confounding factors, MBD > 12.4 Gy increased the risk of EOSL.

\subsection{Early onset of severe lymphopenia and survival}

With a median follow-up of 26.2 (IQR 16.2-39.0) months, the estimated 2-year OS and PFS for all patients were $82.0 \%$ and $76.4 \%$, respectively. Patients with EOSL had decreased OS (2-yr $75.1 \%$ vs. $91.1 \%$, HR $[95 \% \mathrm{CI}]=3.35$ [1.12-9.99], $P=0.021$, Fig. 7A) and PFS (2-yr $71.2 \%$ vs. $83.7 \%$, HR $[95 \% \mathrm{CI}]=2.18$ [0.92-5.21], $P=0.071$, Fig. 7B) than those without EOSL.

Under univariate analysis, EOSL $(P=0.030), \mathrm{MBD}$ ( $P=0.001)$, concurrent cisplatin cycles $(P=0.049)$, pre-RT absolute neutrophil count (ANC) $(P=0.047)$, and pre-RT HGB $(P=0.004)$ were associated with OS. Multivariate COX regression analysis with all factors with $P<0.05$ on univariate analysis showed that EOSL was independently significant for poor OS (HR 3.845 , 95\%CI $1.196-12.360, P=0.024)$. Other adjusting factors including $\mathrm{MBD}(P=0.007)$, concurrent cisplatin cycles $(P=0.007)$, pre-RT ANC $(P=$ $0.016)$, and pre-RT HGB $(P=0.010)$ were also predictive of OS in the final multivariate COX model (Table 2). The C-index and 2-year survival AUC of this multivariate COX model for OS prediction was 0.835 (95\%CI 0.75-0.92) and 0.872, respectively (Fig. 8).

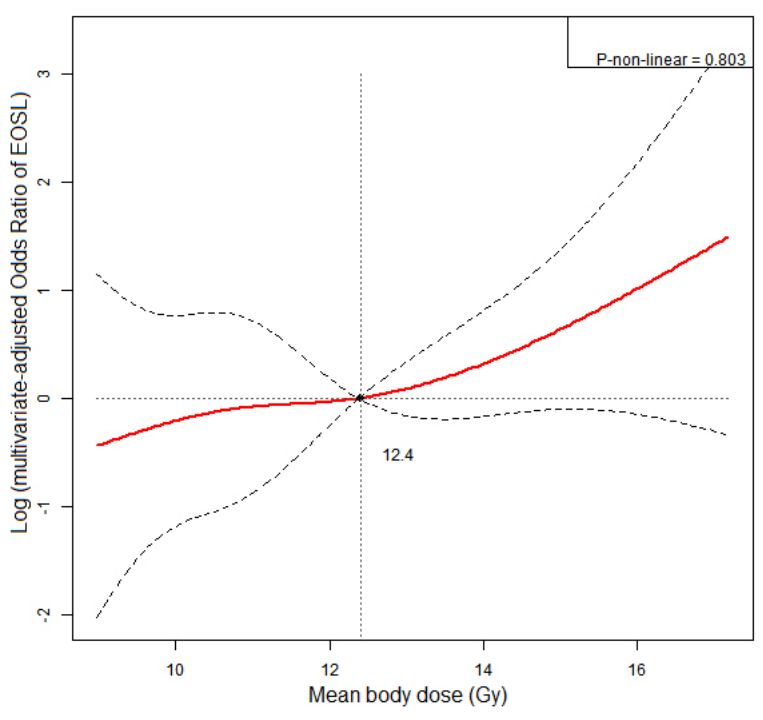

Fig. 6. Restricted cubic spline (RCS) model to illustrate dose-response relationship between mean body dose (MBD) and risk of early onset of severe lymphopenia (EOSL). The dashed lines represented the $95 \%$ confidence intervals. MBD was linearly correlated with risk of EOSL.

\section{Discussion}

Previous studies showed that lymphopenia during RT was common and correlated with survival in patients with cervical cancer $[8,9,12]$. The current study confirmed that the incidence rate of severe lymphopenia was as high as $95.2 \%$ in cervical cancer patients treated with definitive (chemo)RT, which was much higher than those observed in other solid tumors [7], such as brain tumors (20-40\%), head and neck cancers (48-71\%), and thoracic malignancies (40-50\%). Therefore investigating lymphopenia and seeking ways to minimize the risk and severity of lymphopenia in cervical cancer are clinically important topics.

In this study the authors demonstrated that the median time of FOSL was 20 days (about 3 weeks) from the start of RT. Patients with EOSL had decreased OS (2-yr $75.1 \%$ vs. $91.1 \%, P=0.021)$ and PFS (2-yr $71.2 \%$ vs. $83.7 \%, P=0.071)$ than those without EOSL. EOSL was prognostic for poor OS (HR 3.845, 95\%CI 1.196-12.360, $P=0.024$ ) after adjusting factors including MBD, concurrent cisplatin cycles, pre-RT ANC and pre-RT HGB in a multivariate COX model. This OS prediction model had good performance with $\mathrm{C}$-index of 0.835 and AUC of 0.872 , which may be used to guide personized treatment or clinical trial design. In practice, when grade 3-4 lymphopenia was observed at the first 3 weeks of RT, measures such as adopting adaptive RT, 
Table 2

Factors associated with the overall survival for all 104 patients

\begin{tabular}{|c|c|c|c|c|}
\hline \multirow[t]{2}{*}{ Variable } & \multicolumn{2}{|c|}{ Univariate analysis } & \multicolumn{2}{|c|}{ Multivariate analysis } \\
\hline & $\operatorname{HR}(95 \% \mathrm{CI})$ & $P$ & HR $(95 \% \mathrm{CI})$ & $P$ \\
\hline Age $(>60 / \leqslant 60$ years $)$ & $0.338(0.099-1.147)$ & 0.082 & NI & \\
\hline $\operatorname{ECOG}(2 / 0-1)$ & $2.024(0.679-6.040)$ & 0.206 & NI & \\
\hline FIGO stage (IIIA-IVA/ IB1-IIB) & $1.945(0.654-5.786)$ & 0.232 & NI & \\
\hline EBRT technique (Rapidarc/3D-CRT) & $1.080(0.421-2.768)$ & 0.873 & NI & \\
\hline Concurrent cisplatin (Yes/ No) & $1.106(0.317-3.852)$ & 0.875 & NI & \\
\hline Concurrent cisplatin cycles $(5-6 / 0-4)$ & $0.416(0.174-0.995)$ & 0.049 & $0.245(0.088-0.679)$ & 0.007 \\
\hline Induction chemotherapy (Yes/ No) & $1.665(0.480-5.771)$ & 0.421 & NI & \\
\hline Pre-RT WBC (Per $\left.10^{9} / \mathrm{L}\right)$ & $1.072(0.979-1.174)$ & 0.135 & $\mathrm{NI}$ & \\
\hline Pre-RT ANC (Per $\left.10^{9} / \mathrm{L}\right)$ & $1.095(1.001-1.198)$ & 0.047 & $1.115(1.020-1.218)$ & 0.016 \\
\hline Pre-RT ALC (Per $\left.10^{9} / \mathrm{L}\right)$ & $0.430(0.184-1.001)$ & 0.050 & $\mathrm{NI}$ & \\
\hline Pre-RT HGB (Per g/L) & $0.965(0.943-0.988)$ & 0.004 & $0.964(0.938-0.991)$ & 0.010 \\
\hline Pre-RT PLT (Per $\left.10^{9} / \mathrm{L}\right)$ & $1.002(0.998-1.005)$ & 0.316 & NI & \\
\hline Pre-RT AMC (Per $\left.10^{9} / \mathrm{L}\right)$ & $0.821(0.048-14.145)$ & 0.892 & & \\
\hline Mean body dose (Per Gy) & $1.259(1.094-1.449)$ & 0.001 & $1.254(1.065-1.477)$ & 0.007 \\
\hline EOSL (Yes/ No) & $3.351(1.124-9.991)$ & 0.030 & $3.845(1.196-12.360)$ & 0.024 \\
\hline
\end{tabular}

ECOG, Eastern Cooperative Oncology Group; FIGO, Federation of Gynecology and Obstetrics; EBRT, external beam radiotherapy; 3D-CRT, three-dimensional conformal radiotherapy; RT, radiotherapy; WBC, white blood cell; ANC, absolute neutrophil count; ALC, absolute lymphocyte count; HGB, hemoglobin; PLT, platelet; AMC, absolute monocyte count; EOSL, early onset of severe lymphopenia; HR, hazard ratio; CI, confidence interval; NI, not included.
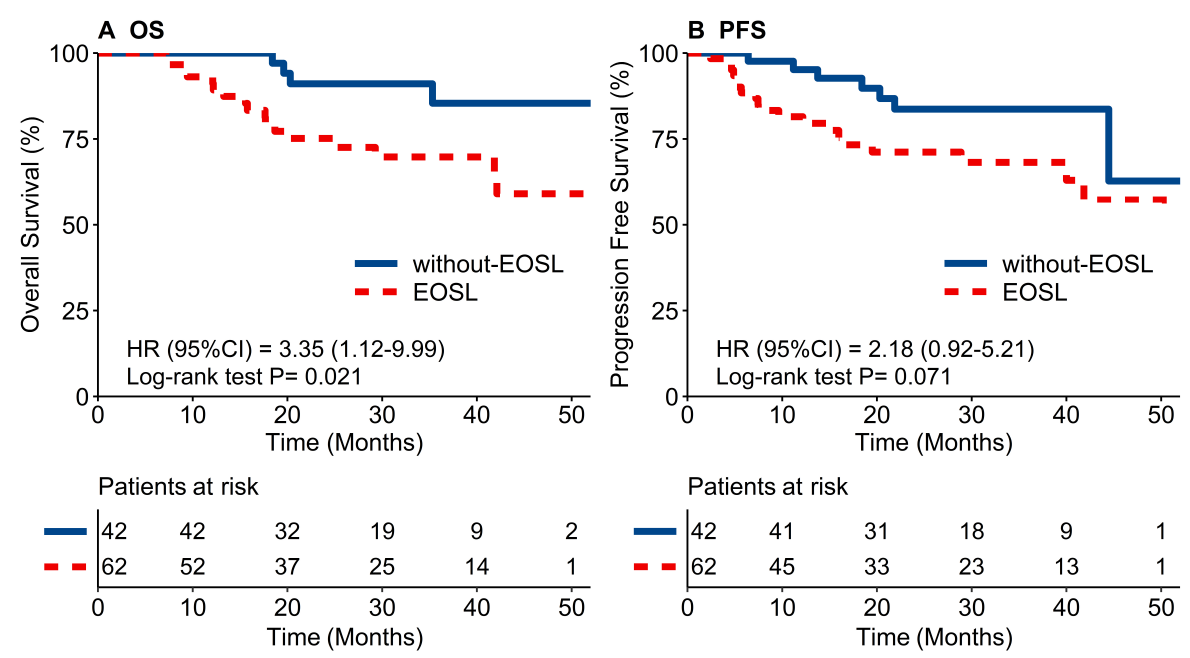

Fig. 7. Kaplan-Meier curves of overall survival (OS) and progression-free survival (PFS). A. OS curves of patients with or without EOSL during radiotherapy (RT) $(2-\mathrm{yr} 75.1 \%$ vs. $91.1 \%, P=0.021)$. B. PFS curves of patients with or without EOSL during RT $(2-\mathrm{yr} 71.2 \%$ vs. $83.7 \%, P=$ $0.071)$.

could be made to preserve or reserve ALC, with the aim to improve treatment outcome.

Lymphocytes are extremely sensitive to radiation, $\mathrm{LD}_{50}$ of lymphocyte (lethal dose required to reduce the surviving fraction of lymphocytes by $50 \%$ ) is only 2 Gy and $\mathrm{LD}_{90}$ is only $3 \mathrm{~Gy}$, exposure to even low doses of radiation can lead to a decrease in the number of peripheral ALC [13]. Even low radiation doses absorbed by the whole body may have a role in the radiationinduced lymphopenia. It was reported that body integral dose was significant predictor of post-treatment lymphocytes in lung cancer [14]. Higher body dosevolume parameters (body V5, V10) may be predictive of severe lymphopenia after palliative RT [15]. In a study on esophageal cancer treated with CCRT, MBD was significantly correlated with lymphocyte nadir during treatment [16]. While our findings were in agreement with the results of previous studies, this study demonstrated a linear relationship ( $P$ for non-linearity $=0.803$ ) between MBD and risk of EOSL and MBD $>12.4$ Gy increased the risk of EOSL. In our opinion, $\mathrm{MBD}$ is a robust dosimetric parameter for radiation- 
2-year Survival AUC $=0.872$

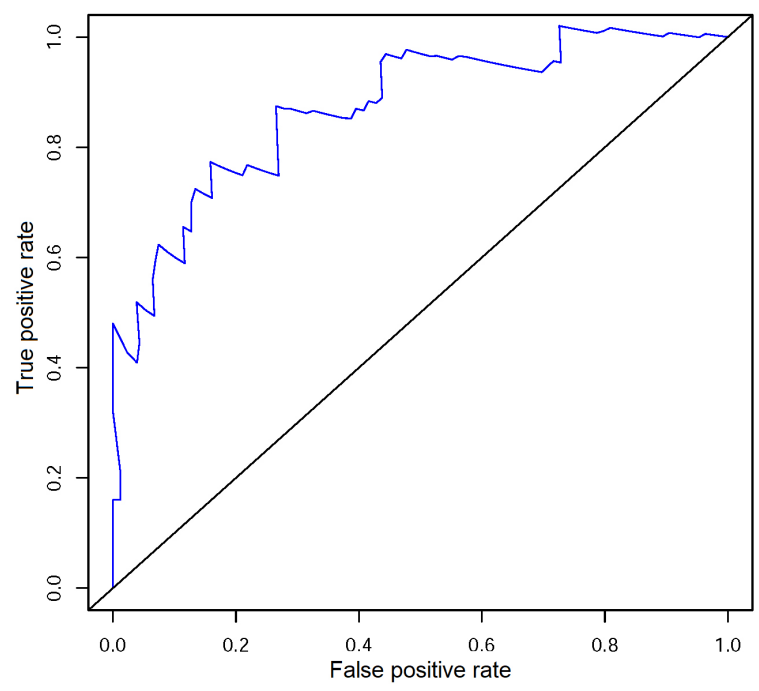

Fig. 8. Receiver operator characteristic (ROC) curve of the multivariate COX model for OS prediction.

induced lymphopenia prediction as it encompasses the effects of both the low dose region and the high dose volume and can be used as an optimization parameter when designing treatment plan.

Although we successfully demonstrated significant association between MBD and risk of EOSL, higher MBD was independently associated with poor OS (HR $1.254,95 \%$ CI $1.065-1.477, P=0.007$ ) in multivariate COX model. We postulated the following reasons for this result: First, FIGO stage was not included in the final COX model of this study. We further found that patients with FIGO IIIA-IVA had higher MBD than those with FIGO IB-II (13.2 vs $11.7 \mathrm{~Gy}, P=0.006)$. Higher MBD partly represented more advanced tumor stage. Second, the effect of pelvic radiation on lymphocyte subgroups was different [17]. Different lymphocyte subsets may have different impacts on treatment outcomes of cervical cancer patients $[18,19]$. Therefore, MBD may impact survival by means of inducing lymphopenia or affecting different lymphocyte subsets. MBD may be a good candidate marker for OS prediction as it correlated with both tumor burden (FIGO stage) and host immune function (EOSL).

The role of chemotherapy on lymphopenia has not been fully investigated. In a study in patients treated with IC for advanced ovarian cancer, ALC increased significantly post-IC compared with the pre-IC values $(P=0.009)$; pre-IC lymphopenia was observed in 27 patients $(40 \%)$, whereas only 16 patients $(24 \%)$ displayed lymphopenia post-IC $(P=0.020)$ [20]. How- ever, in non-small cell lung cancer (NSCLC) patients treated with IC the ALC did not change after two cycles of IC [21]. The current study demonstrated that IC did not correlate with EOSL, however, patients with concurrent cisplatin were more likely to have EOSL. A previous study evaluated effects of platinum compounds on lymphocyte proliferation in vitro and demonstrated that certain platinum salts affected lymphocyte proliferation [22]. The effect of chemotherapy on lymphocyte might be less serious but more complex than that of RT as the cytotoxic mechanisms are diverse among different chemotherapy drugs. It is worthwhile to study the effect of chemotherapy on immune function to assist in chemotherapy choice.

Neutrophils are important components of immune system as well. Contrary to lymphocytes, neutrophils were found to be negatively correlated with survival for patients with cancers. Wisdom et al. showed that neutrophils promoted tumor resistance to RT [23]. High ANC during CCRT was associated with poor local control and survival in cervical cancer patients [23]. In NSCLC, high baseline ANC was a significant survival covariate (HR 1.07, 95\% CI 1.02-1.11, $P=$ 0.017) [24]. This study showed that higher pre-RT ANC was associated with poor OS (HR 1.115, 95\%CI 1.020 $1.218, P=0.016$ ), which was consistent with previous studies.

This study had some limitations. First, the retrospective nature of the study created discordance on the timing of blood test, confounding the interpretation of the results. Second, lymphocyte subtypes had different roles in the treatment outcomes of RT $[18,19]$. However we did not test lymphocyte subtypes and did not collect patients' blood for future research routinely in clinical practice, so there was lack of data on the lymphocyte subtype changes and their impact on the treatment outcome in our patients. Third, we did not exam the relationship of dose to immune organ at risk (OAR) and EOSL, which might have important role in radiationinduced lymphopenia. Finally, the absence of a validation cohort was also a limitation of our study. However, we have demonstrated some interesting results with a linear association between MBD and risk of EOSL and the prognostic effect of EOSL on survival.

In conclusion, our study demonstrated that EOSL during definitive RT correlated with MBD and predicted poor survival in patients with cervical cancer. There are several questions remain to be answered, such as is radiation related lymphopenia truly a driver of inferior survival? Efforts to differentiate this from other contributing factors are required. Furthermore, additional 
research to explore treatment approaches that preserve or restore ALC during definitive RT will be important to improve the treatment outcome of cervical cancer patients with the guidance of these results.

\section{Acknowledgments}

The authors acknowledge Dr. Weiwei Chen for useful discussion.

\section{Author contributions:}

Li Yang: Primary data collection, data analysis, manuscript writing and study design and manuscript approval.

Zhiyuan Xu: Corresponding Author, data collection and double check, data analysis, result interpretation, study design and manuscript approval.

Lingyu Ma: Data double check, data analysis, result interpretation, and manuscript approval.

Qin Liu and Amy TY Chang: Manuscript edit and manuscript approval.

Qian Wang, Jiandong Zha, Jinliang Zhang, Xiaoqin Jiang, Jingjing Zhang: data collection and patient follow-up and manuscript approval.

Feng-Ming (Spring) Kong: Study design, data analysis, result interpretation, manuscript edits and approval. Linlang Guo: Corresponding Author, study design oversight, data quality control, results check and manuscript approval.

\section{Funding}

This project was supported by the grants from Health Commission of Guangdong Province, China (Grant No.: B2020100); HKUSZH Seed Funding, High LevelHospital Program, Health Commission of Guangdong Province, China (Grant No.: HKUSZH201902031); Shenzhen Key Medical Discipline Construction Fund (Grant No.: SZXK014); and Shenzhen Science and Technology Program (Grant NO.: KQTD201804111 85028798).

\section{Conflict of interest}

No.

\section{References}

[1] H. Sung, J. Ferlay, R.L. Siegel, M. Laversanne, I. Soerjo- mataram, A. Jemal and F. Bray, Global Cancer Statistics 2020: GLOBOCAN estimates of incidence and mortality worldwide for 36 Cancers in 185 countries, CA Cancer J Clin 71 (2021), 209-249.

[2] W.J. Koh, N.R. Abu-Rustum, S. Bean, K. Bradley, S.M. Campos, K.R. Cho, H.S. Chon, C. Chu, R. Clark, D. Cohn, M.A. Crispens, S. Damast, O. Dorigo, P.J. Eifel, C.M. Fisher, P. Frederick, D.K. Gaffney, E. Han, W.K. Huh, J.R. Lurain, A. Mariani, D. Mutch, C. Nagel, L. Nekhlyudov, A.N. Fader, S.W. Remmenga, R.K. Reynolds, T. Tillmanns, S. Ueda, E. Wyse, C.M. Yashar, N.R. McMillian and J.L. Scavone, Cervical cancer, version 3.2019, NCCN clinical practice guidelines in oncology, J Natl Compr Canc Netw 17 (2019), 64-84.

[3] M.N. Brodeur, R. Dejean, M.C. Beauchemin, V. Samouëlian, B. Cormier, O.M. Bacha, T. Warkus and M. Barkati, Oncologic outcomes in the era of modern radiation therapy using FIGO 2018 staging system for cervical cancer, Gynecol Oncol 162 (2021), 277-283.

[4] D. Mittal, M.M. Gubin, R.D. Schreiber and M.J. Smyth, New insights into cancer immunoediting and its three component phases-elimination, equilibrium and escape, Curr Opin Immunol 27 (2014), 16-25.

[5] M.H. Melis, K.L. Simpson, S.J. Dovedi, A. Welman, M. MacFarlane, C. Dive, J. Honeychurch and T.M. Illidge, Sustained tumour eradication after induced caspase- 3 activation and synchronous tumour apoptosis requires an intact host immune response, Cell Death Differ 20 (2013), 765-773.

[6] A. Ko, A. Kanehisa, I. Martins, L. Senovilla, C. Chargari, D. Dugue, G. Mariño, O. Kepp, M. Michaud, J.L. Perfettini, G. Kroemer and E. Deutsch, Autophagy inhibition radiosensitizes in vitro, yet reduces radioresponses in vivo due to deficient immunogenic signalling, Cell Death Differ 21 (2014), 92-99.

[7] B.P. Venkatesulu, S. Mallick, S.H. Lin and S. Krishnan, A systematic review of the influence of radiation-induced lymphopenia on survival outcomes in solid tumors, Crit Rev Oncol Hematol 123 (2018), 42-51.

[8] O. Cho, M. Chun, S.J. Chang, Y.T. Oh and O.K. Noh, Prognostic value of severe lymphopenia during pelvic concurrent chemoradiotherapy in cervical cancer, Anticancer Res 36 (2016), 3541-3547.

[9] E.S. Wu, T. Oduyebo, L.P. Cobb, D. Cholakian, X. Kong, A.N. Fader, K.L. Levinson, E.J. Tanner, 3rd, R.L. Stone, A. Piotrowski, S. Grossman and K.L. Roche, Lymphopenia and its association with survival in patients with locally advanced cervical cancer, Gynecol Oncol 140 (2016), 76-82.

[10] X. Han, Q. Yang, J. Zhang and J. Cao, Correlation between changes in the number of peripheral blood lymphocytes and survival rate in patients with cervical cancer after radiochemotherapy, Cancer Radiother 25 (2021), 72-76.

[11] J.H. Joo, S.Y. Song, J. Park, E.K. Choi, S.Y. Jeong and W. Choi, Lymphocyte depletion by radiation therapy alone is associated with poor survival in non-small cell lung cancer, International Journal of Radiation Oncology Biology Physics 96 (2016), E478.

[12] L. Yang, Z.Y. Xu, A.T. Chang, Q. Wang, X. Chen, L. Shen, S.K. Hui, K.W. Lee, W. Chan, Y. Zhou, F. Chen, J. Zha, J.Y. Jin and F.M. Kong, A potential survival impact of blood immune cells in patients with cervical carcinoma treated with concurrent chemoradiotherapy, International Journal of Radiation Oncology Biology Physics 105 (2019), E343.

[13] N. Nakamura, Y. Kusunoki and M. Akiyama, Radiosensitivity of CD4 or CD8 positive human T-lymphocytes by an in vitro colony formation assay, Radiat Res 123 (1990), 224-227.

[14] N. Joseph, A. McWilliam, J. Kennedy, K. Haslett, J. Mahil, 
A. Gavarraju, H. Mistry, M. Van Herk, C. Faivre-Finn and A. Choudhury, Post-treatment lymphocytopaenia, integral body dose and overall survival in lung cancer patients treated with radical radiotherapy, Radiother Oncol 135 (2019), 115-119.

[15] T. Saito, R. Toya, T. Matsuyama, A. Semba and N. Oya, Dosimetric predictors of treatment-related lymphopenia induced by palliative radiotherapy: predictive ability of dose-volume parameters based on body surface contour, Radiol Oncol $\mathbf{5 1}$ (2017), 228-234

[16] R. Davuluri, W. Jiang, P. Fang, C. Xu, R. Komaki, D.R. Gomez, J. Welsh, J.D. Cox, C.H. Crane, C.C. Hsu and S.H. Lin, Lymphocyte nadir and esophageal cancer survival outcomes after chemoradiation therapy, Int J Radiat Oncol Biol Phys 99 (2017), 128-135.

[17] G.P. Swanson, S.G. Jhavar and K. Hammonds, The effect of pelvic radiation alone on lymphocyte subgroups, Clin Transl Radiat Oncol 23 (2020), 100-102.

[18] E.S. Jordanova, A. Gorter, O. Ayachi, F. Prins, L.G. Durrant, G.G. Kenter, S.H. van der Burg and G.J. Fleuren, Human leukocyte antigen class I, MHC class I chain-related molecule $\mathrm{A}$, and $\mathrm{CD} 8+/$ regulatory $\mathrm{T}$-cell ratio: which variable determines survival of cervical cancer patients? Clin Cancer Res 14 (2008), 2028-2035

[19] M. Ozsahin, N.E. Crompton, S. Gourgou, A. Kramar, L. Li, Y. Shi, W.J. Sozzi, A. Zouhair, R.O. Mirimanoff and D. Azria CD4 and CD8 T-lymphocyte apoptosis can predict radiationinduced late toxicity: a prospective study in 399 patients, Clin Cancer Res 11 (2005), 7426-7433.
[20] Y. Yoshino, A. Taguchi, M. Takao, T. Kashiyama, A. Furusawa, M. Uno, S. Okada, N. Kino and T. Yasugi, Lymphopenia after induction chemotherapy correlates with incomplete surgical resection in patients with advanced ovarian cancer, Int J Clin Oncol 24 (2019), 428-436.

[21] J.L. Campian, X. Ye, M. Brock and S.A. Grossman, Treatmentrelated lymphopenia in patients with stage III non-small-cell lung cancer, Cancer Invest 31 (2013), 183-188.

[22] M. Di Gioacchino, L. Di Giampaolo, N. Verna, M. Reale, M.B. Di Sciascio, A.R. Volpe, M. Carmignani, J. Ponti, R. Paganelli, E. Sabbioni and P. Boscolo, In vitro effects of platinum compounds on lymphocyte proliferation and cytokine release, Ann Clin Lab Sci 34 (2004), 195-202.

[23] A.J. Wisdom, C.S. Hong, A.J. Lin, Y. Xiang, D.E. Cooper, J. Zhang, E.S. Xu, H.C. Kuo, Y.M. Mowery, D.J. Carpenter, K.T. Kadakia, J.E. Himes, L. Luo, Y. Ma, N. Williams, D.M. Cardona, M. Haldar, Y. Diao, S. Markovina, J.K. Schwarz and D.G. Kirsch, Neutrophils promote tumor resistance to radiation therapy, Proc Natl Acad Sci U S A 116 (2019), 18584-18589.

[24] A. Salem, H. Mistry, A. Backen, C. Hodgson, P. Koh, E. Dean, L. Priest, K. Haslett, I. Trigonis, A. Jackson, M.C. Asselin, C. Dive, A. Renehan, C. Faivre-Finn and F. Blackhall, Cell death, inflammation, tumor burden, and proliferation blood biomarkers predict lung cancer radiotherapy response and correlate with tumor volume and proliferation imaging, Clin Lung Cancer 19 (2018), 239-248e7. 